\title{
Calcium Alginate and Calcium Alginate-Chitosan Beads Containing Celecoxib Solubilized in a Self-Emulsifying Phase
}

\author{
Lorena Segale, Lorella Giovannelli, Paolo Mannina, and Franco Pattarino \\ Dipartimento di Scienze del Farmaco, Università del Piemonte Orientale, Largo Donegani 2, 28100 Novara, Italy \\ Correspondence should be addressed to Lorena Segale; lorena.segale@uniupo.it
}

Received 4 December 2015; Revised 25 February 2016; Accepted 9 March 2016

Academic Editor: Vitor M. Correlo

Copyright (C) 2016 Lorena Segale et al. This is an open access article distributed under the Creative Commons Attribution License, which permits unrestricted use, distribution, and reproduction in any medium, provided the original work is properly cited.

\begin{abstract}
In this work alginate and alginate-chitosan beads containing celecoxib solubilized into a self-emulsifying phase were developed in order to obtain a drug delivery system for oral administration, able to delay the drug release in acidic environment and to promote it in the intestinal compartment. The rationale of this work was linked to the desire to improve celecoxib therapeutic effectiveness reducing its gastric adverse effects and to favor its use in the prophylaxis of colon cancer and as adjuvant in the therapy of familial polyposis. The systems were prepared by ionotropic gelation using needles with different diameters (400 and $600 \mu \mathrm{m})$. Morphology, particle size, swelling behavior, and in vitro drug release performance of the beads in aqueous media with different $\mathrm{pH}$ were investigated. The experimental results demonstrated that the presence of chitosan in the formulation caused an increase of the mechanical resistance of the bead structure and, as a consequence, a limitation of the bead swelling ability and a decrease of the drug release rate at neutral $\mathrm{pH}$. Alginate-chitosan beads could be a good tool to guarantee a celecoxib colon delivery.
\end{abstract}

\section{Introduction}

Drug delivery systems containing biodegradable natural polymers are the object of more and more research studies considering the advantages that these materials can offer [1]. Among them, those containing alginate and chitosan have been widely exploited in pharmaceutical field [2-4].

Alginate is a water soluble natural biopolymer extracted from brown algae and composed of alternating blocks of 1-4 $\alpha$-L-guluronic and $\beta$-D-mannuronic acid residues [5]. This polymer forms hydrogels in presence of divalent cations like $\mathrm{Ca}^{2+}, \mathrm{Ba}^{2+}, \mathrm{Sr}^{2+}$, and $\mathrm{Zn}^{2+}[6,7]$ and this characteristic allows preparing drug loaded beads [8]. The mechanism of this gelation process involves guluronic residues with the specific chelation of $\mathrm{Ca}^{2+}$ forming the so-called "egg-box" structure [9]. Many researchers focused their attention on the development of calcium alginate beads as controlled drug delivery systems for the oral administration of drug molecules and proteins [10-12].

Chitosan is a biocompatible, biodegradable, nontoxic, linear polysaccharide composed of D-glucosamine and $\mathrm{N}$ acetyl-D-glucosamine units linked by $\beta-(1-4)$ glycosidic linkages [13]. Chitosan can be derived by partial deacetylation of chitin from crustacean shells and it is widely used for cell culture, drug delivery, and food additives [14-16].

Cross-linking of alginate and chitosan in a hydrogel is used to provide materials useful for medical and pharmaceutical applications; the obtained systems are characterized by enhanced stability compared to those obtained with a single polymer $[17,18]$. In controlled drug delivery alginatechitosan polyelectrolyte complex has received much attention in recent years [12, 19-22]. The two polymers form the polyelectrolyte complex via the ionic interaction between the carboxyl residues of alginate and the amino residues of chitosan. The alginate-chitosan beads can be produced by different methods: the two-step procedure and the one-step procedure [23]. In the first one, calcium alginate gel beads are produced by dropping a solution of alginate into a gelling bath containing calcium ions. The resulting beads are then transferred into a chitosan solution to form the membrane on their surface. The one-step procedure requires that the droplets of alginate solution fall into an aqueous solution containing both the gelling agent for alginate (e.g., calcium ions) and chitosan [24]. The choice of the production method 
TABLE 1: Composition of calcium alginate and calcium alginate-chitosan beads.

\begin{tabular}{|c|c|c|c|c|}
\hline Formulation & Sodium alginate $(\% \mathrm{w} / \mathrm{w})$ & Self-emulsifying phase $(\% \mathrm{w} / \mathrm{w})$ & Gelling bath & Needle diameter $(\mu \mathrm{m})$ \\
\hline \multirow{3}{*}{ Cal 600} & \multirow{3}{*}{1.5} & Celecoxib 27.4 & \multirow{3}{*}{$\mathrm{CaCl}_{2} 100 \mathrm{mM}$} & \multirow{3}{*}{600} \\
\hline & & Labrasol 68.5 & & \\
\hline & & TPGS 4.1 & & \\
\hline \multirow{3}{*}{ Cal 400} & \multirow{3}{*}{1.5} & Celecoxib 27.4 & \multirow{3}{*}{$\mathrm{CaCl}_{2} 100 \mathrm{mM}$} & \multirow{3}{*}{400} \\
\hline & & Labrasol 68.5 & & \\
\hline & & TPGS 4.1 & & \\
\hline \multirow{3}{*}{ CAlCh 600} & \multirow{3}{*}{1.5} & Celecoxib 27.4 & \multirow{3}{*}{$\mathrm{CaCl}_{2} 100 \mathrm{mM}+0.2 \%$ chitosan } & \multirow{3}{*}{600} \\
\hline & & Labrasol 68.5 & & \\
\hline & & TPGS 4.1 & & \\
\hline \multirow{3}{*}{ CAlCh 400} & \multirow{3}{*}{1.5} & Celecoxib 27.4 & \multirow{3}{*}{$\mathrm{CaCl}_{2} 100 \mathrm{mM}+0.2 \%$ chitosan } & \multirow{3}{*}{400} \\
\hline & & Labrasol 68.5 & & \\
\hline & & TPGS 4.1 & & \\
\hline
\end{tabular}

is responsible for the properties of the beads because of the amount of bound chitosan in the resulting product. At the same time, the characteristics of the beads are affected by the molecular weight of the selected polymers and/or by the percentages of the various residues in the polymeric molecules $[25,26]$.

Celecoxib, a fluorinated benzenesulfonamide derivative, is a nonsteroidal anti-inflammatory drug (NSAID) with a highly selective cyclooxygenase-2 (COX-2) inhibitory action. It possesses anti-inflammatory, analgesic, and antipyretic activities due to the inhibition of prostaglandin synthesis catalyzed by COX-2. Recently, this drug was frequently investigated for its anticancer activity using in vitro and in vivo models [27-30]. Preclinical studies on celecoxib reported prominent anticancer activity against head and neck squamous cell carcinoma, colon cancer, breast cancer, and lung cancer $[27,28]$.

In this work alginate and alginate-chitosan beads containing celecoxib solubilized into a self-emulsifying phase were developed in order to obtain a drug delivery system for oral administration, able to delay the drug release in acidic environment and to promote it in the intestinal compartment. The rationale of this work was linked to the desire to improve celecoxib therapeutic effectiveness reducing its gastric adverse effects and to favor its use in the prophylaxis of colon cancer and as adjuvant in the therapy of familial polyposis [31-33]. The aim of the current study was the evaluation and the comparison of the properties of celecoxib loaded calcium alginate and calcium alginate-chitosan beads. Morphology, particle size, swelling behavior, and in vitro drug release performance of beads in aqueous media with different $\mathrm{pH}$ were investigated.

\section{Materials and Methods}

2.1. Materials. Celecoxib was obtained from Chemos $\mathrm{GmbH}$ (Regenstauf, Germany). Anhydrous calcium chloride and sodium alginate (molecular weight 120000-190000 g/mol; 1.56 mannuronic-guluronic residues ratio) were purchased from Sigma Aldrich (Milan, Italy), while low molecular weight chitosan was from Fluka (Milan, Italy). Labrasol (caprylocaproyl macrogol-8 glycerides) was a gift of Gattefossè (Milan, Italy); TPGS (D- $\alpha$-tocopheryl polyethylenglycol 1000 succinate) was kindly donated by Isochem (Gennevillers, France). All other chemicals were of analytical grade.

2.2. Preparation of Calcium Alginate Beads. Calcium alginate beads were prepared by gelation method using calcium ions as cross-linking agent. In detail, a $1.5 \%(\mathrm{w} / \mathrm{w})$ sodium alginate aqueous solution was mixed with a drug loaded selfemulsifying phase in 4:1 ratio and added drop by drop to a $100 \mathrm{mM} \mathrm{CaCl}_{2}$ solution [34]. The self-emulsifying phase was prepared mixing weighed amounts of Labrasol and TPGS at $50^{\circ} \mathrm{C}$ and dissolving celecoxib in the excipient solution. The emulsion (sodium alginate solution and selfemulsifying phase) was manually extruded in the hardening bath through needles with 400 or $600 \mu \mathrm{m}$ inner diameter, under constant gentle stirring, at room temperature. After 15 minutes, the beads were collected, washed with deionised water to eliminate the excess of calcium ions and then dried at $40^{\circ} \mathrm{C}$ overnight. The composition of prepared formulations, coded CAl 600 and CAl 400, was listed in Table 1.

2.3. Preparation of Calcium Alginate-Chitosan Beads. Calcium alginate-chitosan beads (identified as CAlCh 600 and CAlCh 400) were prepared according to the one step method. The procedure was identical to that adopted in the case of alginate beads with the exception that the hardening bath was a $0.2 \%(\mathrm{w} / \mathrm{w})$ chitosan solution in diluted acetic acid (1\%) containing $\mathrm{CaCl}_{2}$ at a concentration of $100 \mathrm{mM}$. The composition of the chitosan formulations was reported in Table 1.

2.4. Morphological and Particle Size Analysis. The morphology of the wet and dried beads and the particle size of the dried beads were analysed using a Motic SMZ168 stereomicroscope and an image analysis software (Motic Image Plus 2.0). For each formulation, the particle size was calculated as the average value of the size of 20 dried particles. 
2.5. Drug Content. Six milligrams of drug loaded dried beads was solubilized in phosphate buffer solution $(100 \mathrm{~mL})$ at $\mathrm{pH} 6.8$ added of $0.75 \%(\mathrm{w} / \mathrm{v})$ sodium laurylsulphate at $70^{\circ} \mathrm{C}$ under stirring for two hours. After cooling, the obtained solutions were filtrated and analysed spectrophotometrically at $255 \mathrm{~nm}$; the results are the average of at least three determinations.

2.6. Swelling Study. Swelling studies were carried out at $37^{\circ} \mathrm{C}$ on dried beads put into three aqueous media characterized by different $\mathrm{pH}$ : hydrochloric acid at $\mathrm{pH} 1.0$ and phosphate buffer at $\mathrm{pH} 6.8$ and $\mathrm{pH} 7.4$.

Accurately weighed amounts of calcium alginate and calcium alginate-chitosan dried beads were put in glass vials containing $5 \mathrm{~mL}$ of each fluid. After fixed time intervals (5, $15,30,60$, and 120 minutes), the samples were recovered, gently wiped with paper, and weighed again. The dynamic weight change of the beads with respect to time, defined as swelling degree $(\mathrm{Sw})$, was calculated according to the following equation:

$$
\mathrm{Sw} \%=\frac{W_{t}-W_{0}}{W_{0}} \times 100,
$$

where $W_{t}$ is the weight of the beads in the swollen state at time $t$ and $W_{0}$ is the initial weight of the dried beads [35].

2.7. Celecoxib Release Study. The in vitro release studies were performed in hydrochloric acid at $\mathrm{pH} 1.0$ and in phosphate buffer at $\mathrm{pH} 6.8$ and at $\mathrm{pH} 7.4$ added of $0.75 \%$ sodium laurylsulphate to guarantee the maintenance of the sink conditions. The studies were carried out by placing accurately weighed amounts of each formulation, equivalent to $8 \mathrm{mg}$ of celecoxib, in $500 \mathrm{~mL}$ of the selected fluid at $37^{\circ} \mathrm{C}$ under a rotation rate of $100 \mathrm{rpm}$ (apparatus 2, paddle). Filtered samples were withdrawn at specific time intervals without replacement and analysed for celecoxib content using an UV spectrophotometer at $255 \mathrm{~nm}$ when the fluid was $\mathrm{HCl}$ and phosphate buffer at $\mathrm{pH} 6.8$ or at $256 \mathrm{~nm}$ in the case of phosphate buffer at $\mathrm{pH}$ 7.4. Each experiment was done in triplicate.

The drug release performances of calcium alginate and calcium alginate-chitosan beads were compared using the dissolution parameters $t 10 \%, t 50 \%$, and $t 90 \%$ which indicate the time points at which $10 \%, 50 \%$, and $90 \%$ of the drug were released [36] and the $f 2$ similarity parameter [37]. For curves to be considered similar $f 2$ values should be close to 100 , and $f 2$ values greater than $50(50-100)$ ensure sameness or equivalence of the two curves.

2.8. Statistical Analysis. The results were statistically analysed to test significant differences by Student's $t$-test, at $95 \%$ confidence interval; $p$ values less than 0.05 were considered statistically significant.

\section{Results and Discussion}

Alginate and alginate-chitosan beads were obtained by ionotropic gelation method dropping an emulsion, composed of alginate aqueous solution and the drug loading selfemulsifying phase, through $23 \mathrm{G}(600 \mu \mathrm{m})$ or $27 \mathrm{G}(400 \mu \mathrm{m})$ needles, in a calcium chloride or in a calcium chloridechitosan gelling bath. The excipients selected for the selfemulsifying phase were Labrasol, a liquid component with self-emulsifying and solubility enhancer properties, and D- $\alpha$ tocopheryl polyethylenglycol 1000 succinate as coemulsifying and absorption enhancer agent (Table 1). The composition of the self-emulsifying phase was the same used in a previous work [34].

Stereomicroscopic images of wet and dry alginate and alginate-chitosan beads are reported in Figures 1 and 2 . Immediately after the preparation CAl 600 beads (Figure 1(a)) show regular shape and homogeneous dimensions; they are white and opaque with a smooth, glossy, and homogeneous surface.

The drying process does not change the shape of beads but leads to the reduction of their dimensions and modifies the characteristics of their surface, which is irregular and wrinkled (Figure 1(b)). The loss of water induces a decrease of the distance between the polymeric chains and a variation of the structure of the beads, which is not compact and continuous but composed of small micronuclei adhering to each other. The fully swollen CAlCh 600 particles are slightly yellow and quite regular in shape and show a smooth surface (Figure $1(\mathrm{c})$ ). In this case, the drying process affects the spherical shape of the beads (Figure 1(d)); they become ellipsoidal; their dimension decreases and their surface is very rough. Moreover, after drying a partial surface agglomeration of the beads is observed: it is attributable to the adhesive properties of chitosan [38]. In the case of beads prepared using a $400 \mu \mathrm{m}$ needle (Figures 2(a)-2(c)), the images recorded immediately after the preparation give evidence that for both the formulations (with or without chitosan) particles are not homogeneous in diameters even if they are regular in shape. CAl 400 and CAlCh 400 dry beads (Figure 2(b)) have a surface characterized by asperity and concavity; moreover, in the case of alginate-chitosan formulation the particle shape is completely irregular and the presence of solid bridges of chitosan is well evident which bind the beads impeding their separation.

Average size of celecoxib loaded beads is between 715 and $896 \mu \mathrm{m}$ (Table 2). The diameter of the beads was significantly affected by the diameter of the needle used during preparation $(p<0.05)$. The addition of chitosan to alginate beads changes significantly their dimensions only when the $400 \mu \mathrm{m}$ needle was used $(p<0.05)$. Moreover, as indicated by the high values of the standard deviation, using the $400 \mu \mathrm{m}$ needle the final product is a family of particles inhomogeneous in dimensions.

All the formulations contain high amount of drug (Table 2) homogeneously distributed in the excipient matrix and the differences among their drug content were not significant except for CAlCh 400 slightly lower than the others $(p<0.001)$. The percentage of celecoxib in the beads exceeds the theoretical value and this is due to the loss of Labrasol during the curing time [34], justifiable considering the high affinity between this excipient and water which drives it out of the beads into the gelling bath. 


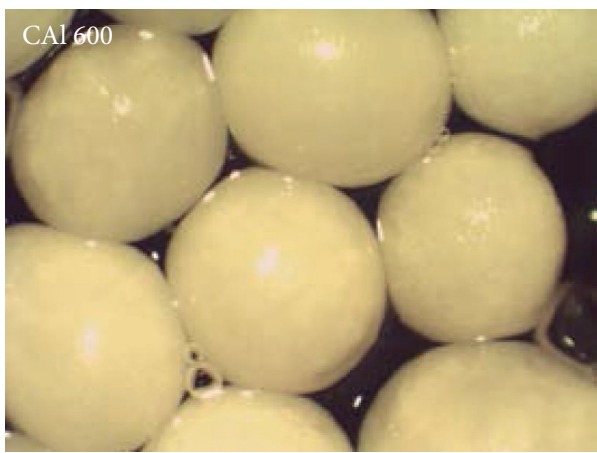

(a)

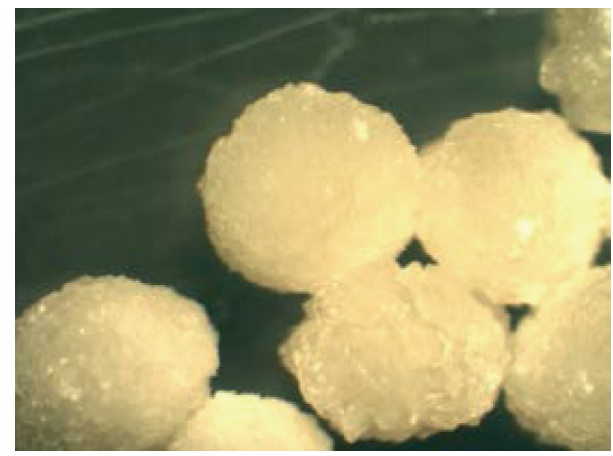

(c)

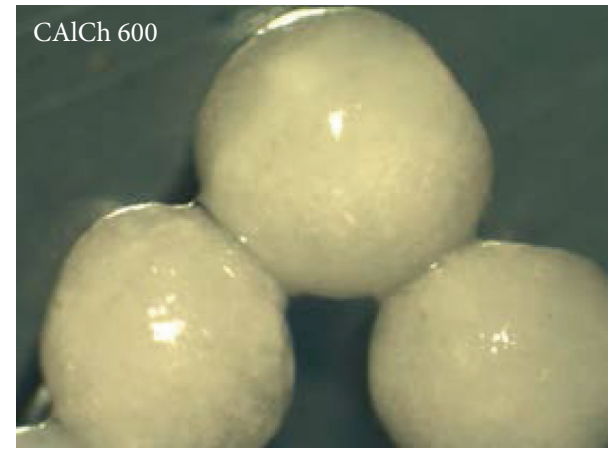

(b)

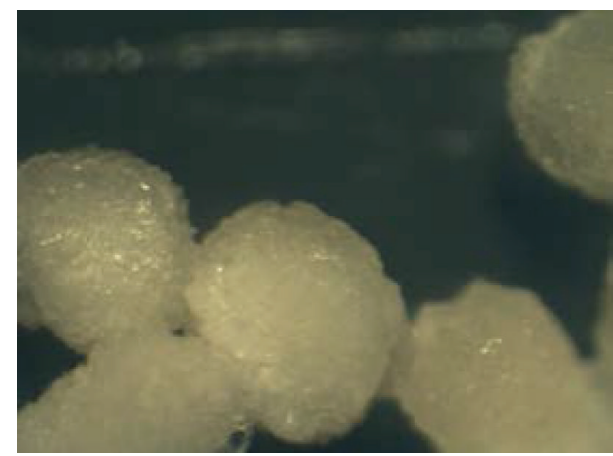

(d)

FIGURE 1: Stereomicroscopic images of wet (a-b) (2x magnification) and dry (c-d) (3x magnification) beads obtained using a needle of $600 \mu \mathrm{m}$ in diameter.

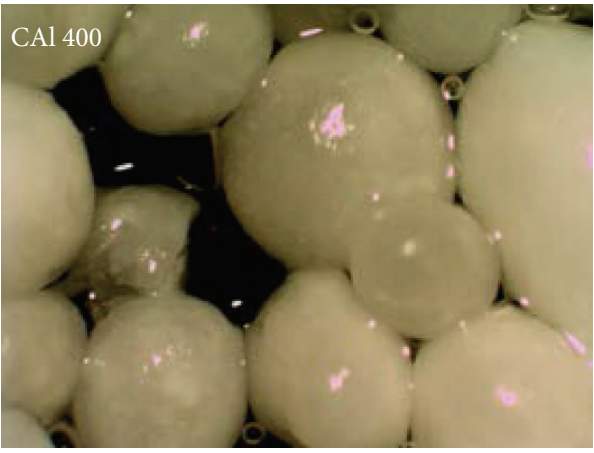

(a)

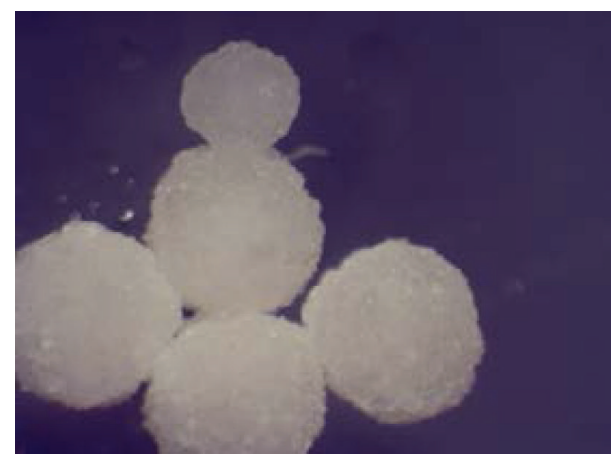

(c)

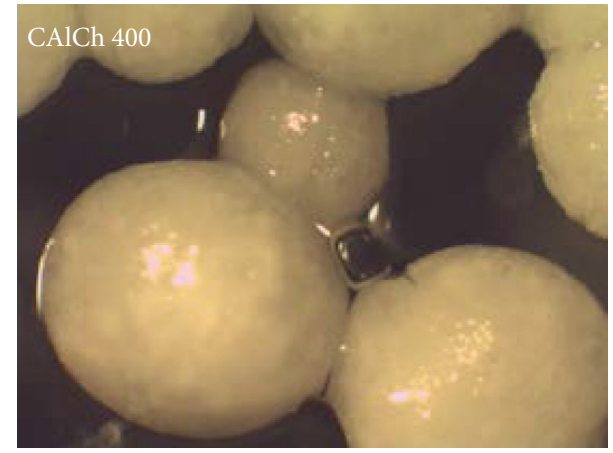

(b)

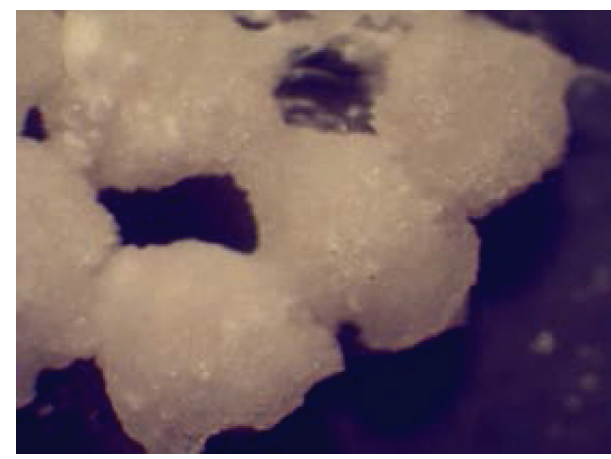

(d)

FIGURE 2: Stereomicroscopic images of wet (a-b) (2x magnification) and dry (c-d) (3x magnification) beads obtained using a needle of $400 \mu \mathrm{m}$ diameter. 
TABLE 2: Average diameter and celecoxib content of the dry beads.

\begin{tabular}{lcc}
\hline & Diameter $(\mu \mathrm{m})$ & Drug content $(\%)$ \\
\hline CAl 600 & $896 \pm 64.24$ & $42.10 \pm 1.30$ \\
CAl 400 & $715 \pm 80.96$ & $43.63 \pm 0.77$ \\
CAlCh 600 & $881 \pm 66.87$ & $40.94 \pm 1.37$ \\
CAlCh 400 & $795 \pm 103.70$ & $39.78 \pm 0.66$ \\
\hline
\end{tabular}

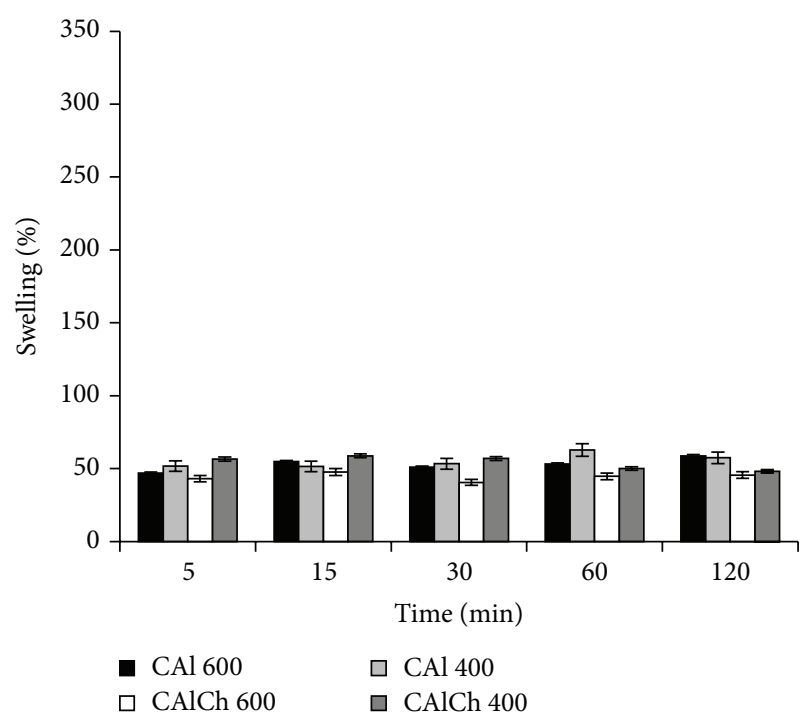

FIGURE 3: Swelling degree in hydrochloric acid at pH 1.0.

A peculiar property of alginate or alginate-chitosan microparticulate systems in the dry form is their ability, after contact with an aqueous fluid, to rehydrate, take up the fluid, and undergo a swelling process, mainly associated with the hydration of the hydrophilic groups of polymers. When the fluid is water, it penetrates into the particles filling the pores among the polymeric chains and causing an important swelling of the system, without erosion/disintegration. Selecting fluids with different $\mathrm{pH}$, the swelling behavior of the beads could change. For this reason, the swelling ability of calcium alginate and of calcium alginate-chitosan beads has been evaluated in $\mathrm{HCl}$ at $\mathrm{pH} 1.0$ and in phosphate buffer solutions at $\mathrm{pH} 6.8$ and 7.4 (Figures 3-5).

In acidic environment (Figure 3), there are no differences in the swelling ability of the four formulations; at this $\mathrm{pH}$, the maximum swelling degree is not over $60 \%$. The alginate and alginate-chitosan beads absorb part of the fluid; their weight initially increases and then remains constant. At this $\mathrm{pH}$, for the alginate systems (CAl 600 and CAl 400), the carboxylate groups of the polymer localized on the surface of particles are protonated and a layer of alginic acid forms. The insolubility of alginic acid in this fluid and the formation of hydrogen bonds, responsible for an increase of the structure stability, impede the penetration of additional fluid into the deeper layers of particles, limiting their swelling. The same swelling behavior is observed for CAlCh 600 and CAlCh 400 systems. Even if, in acidic environment, chitosan is highly soluble and charged for the conversion of its amine units into $\mathrm{NH}^{3+}$

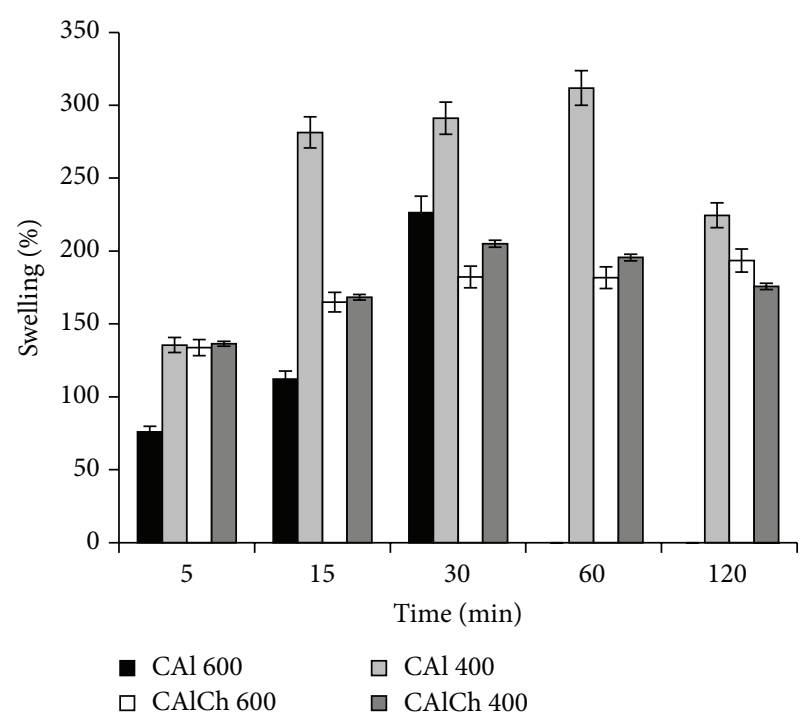

FIGURE 4: Swelling degree in phosphate buffer at pH 6.8 .

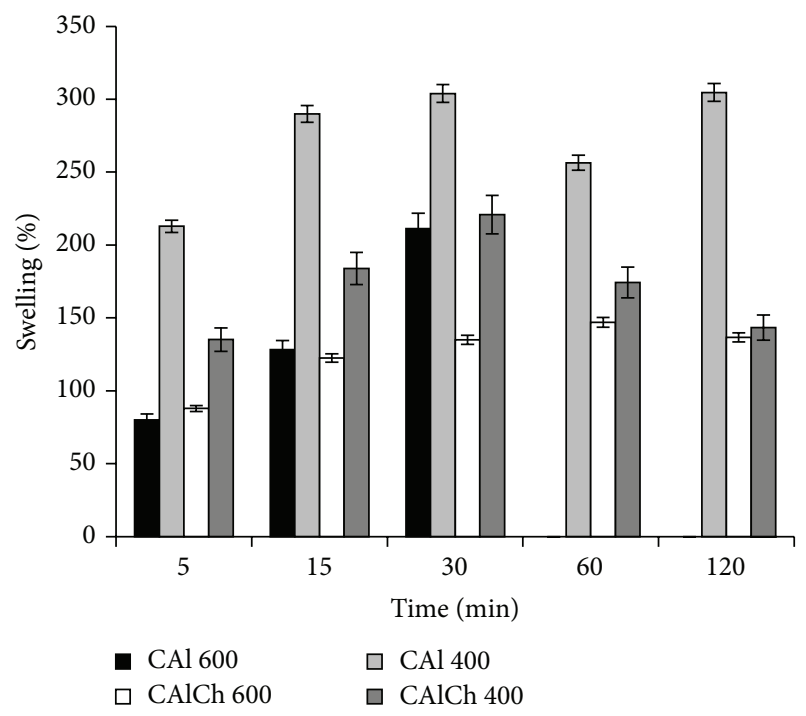

FIGURE 5: Swelling degree in phosphate buffer at pH 7.4.

soluble form, the interaction of amino groups and protonated carboxylic groups is not strong enough to promote swelling. Thus, the limited total swelling behavior is dominated by calcium alginate structure.

Figures 4 and 5 show that the formulations exhibit high swelling ability at $\mathrm{pH} 6.8$ and $\mathrm{pH}$ 7.4. For CAl 600 formulation, the weight of particles grows rapidly, reaches a peak after 30 minutes, and then decreases abruptly because of the erosion/disintegration of the system. This behavior can be due to ion exchange reaction between $\mathrm{Na}^{+}$(present in the phosphate buffer) and $\mathrm{Ca}^{2+}$ linked to carboxylic groups of alginate. Monovalent ions replace bivalent ones causing the breakup of the "egg-box" structure and the increase of the distance between the polymeric chains and favoring the fluid absorption and the swelling of the systems. 


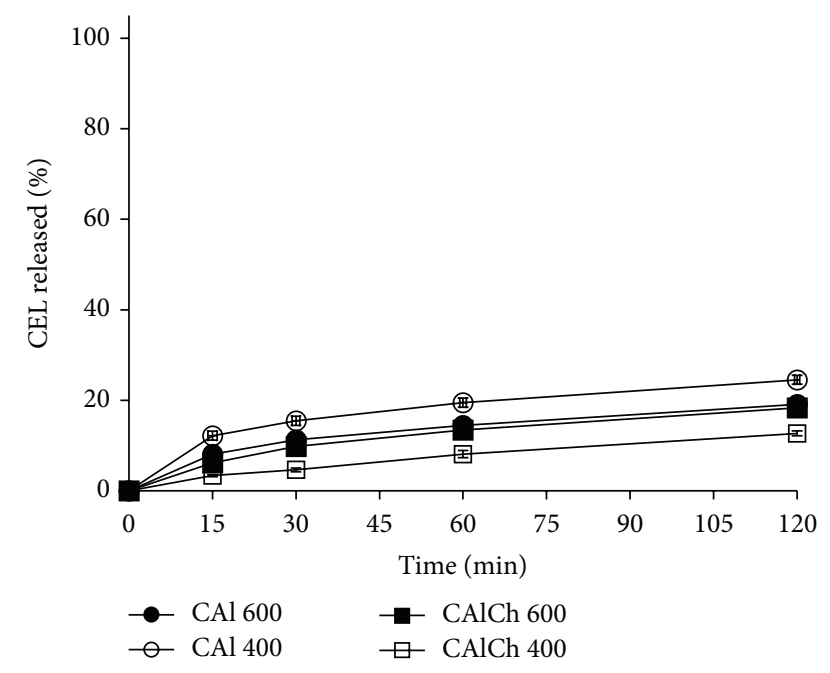

FIgURE 6: Celecoxib release profiles in hydrochloric acid at pH 1.0.

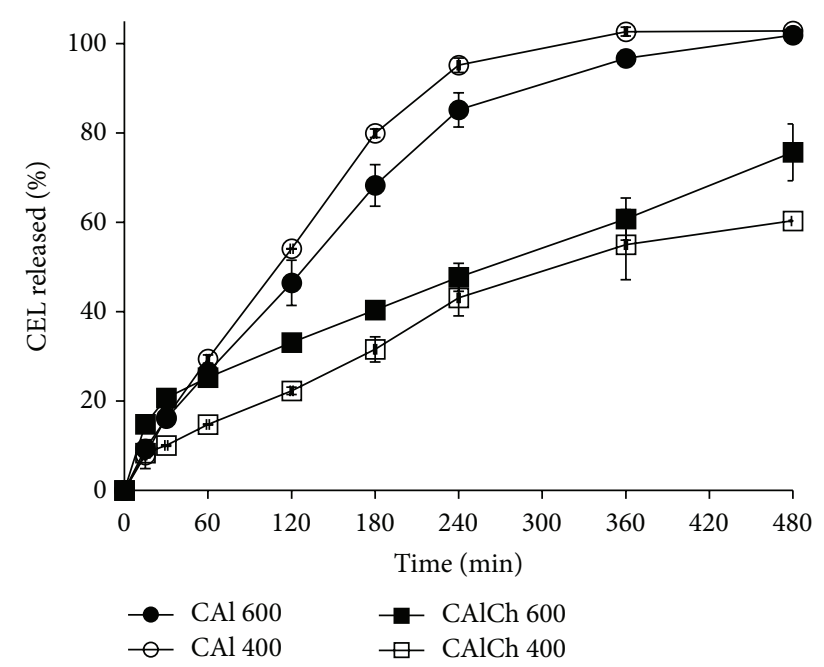

FIGURE 7: Celecoxib release profiles in phosphate buffer at pH 6.8.

This process goes on until the osmotic pressure into the beads balances the strength of the cross-linking bonds and physical entanglements, which preserve the structure of the beads. Thus, particles start to disintegrate and their weight diminishes.

The results obtained from the swelling study evidence that CAlCh 600 and CAlCh 400 beads are characterized by a more resistant structure compared to CAl 600 and CAl 400, probably attributable to the interactions between alginate and chitosan chains. The maximum swelling degree of chitosan beads is lower than that of the alginate; alginate-chitosan systems are able to reach a swelling equilibrium in about 30 minutes and to maintain their weight at a constant level until the end of the test. Probably the interactions between the two polymers are responsible for the formation of particles with a considerable mechanical resistance, which limits the fluid uptake and the structure disintegration. Finally, comparing

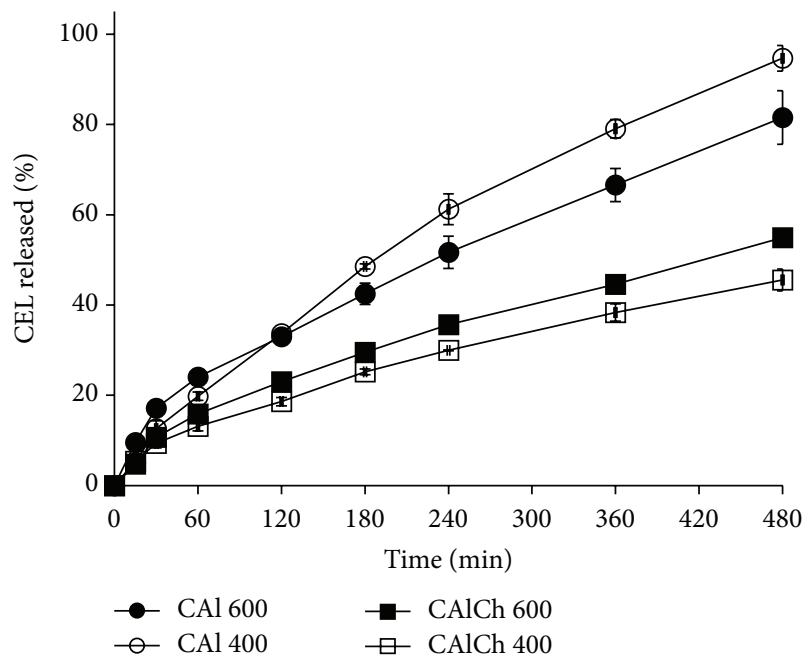

FIGURE 8: Celecoxib release profiles in phosphate buffer at $\mathrm{pH} 7.4$.

the swelling behavior of CAl 400 versus CAl 600 and CAlCh 400 versus CAlCh 600 beads (same composition, different diameter of the needle used in the preparation process), it is possible to note that in phosphate buffers CAl 400 and CAlCh 400 reached a swelling maximum peak higher than that of CAl 600 and CAlCh 600, respectively.

The drug release profiles obtained from the different formulations at $\mathrm{pH}$ of 1.0, 6.8, and 7.4 are shown in Figures $6-8$. The in vitro celecoxib release is affected by the $\mathrm{pH}$ of the selected fluid: the percentage of drug released in acidic medium in two hours is quite low and varies between $12.70 \%$ and $24.53 \%$ (Figure 6). The delay of celecoxib release can be ascribed to the reduced swelling ability of the systems in this fluid; neither the composition of the beads nor their diameter affects the drug release performance ( $f 2$ parameter values always over 50). At $\mathrm{pH} 1.0$, the release process is governed only by the diffusion of the drug. This result allows satisfying the first object of this research work that is to minimize drug delivery in acidic environment to promote and favor this process at intestinal level.

In phosphate buffer at $\mathrm{pH} 6.8$, the formulations are characterized by a drug release behavior affected by their composition and not significantly by their dimensions (Figure 7). In this fluid, the systems initially swell and then erode/disintegrate and, consequently, the drug release process is driven at first by diffusion and then by the polymeric relaxation. Alginate beads (CAl 600 and CAl 400) are able to complete the celecoxib release in about eight hours; on the contrary no more than $75 \%$ of the drug loaded in alginate-chitosan beads passes in solution after the same time. The comparison of the results from alginate and alginatechitosan microparticle formulations reveals that the profiles are not similar having $f 2$ values lower than 50 . A possible explanation for such a behavior is the electrostatic interaction between carboxyl groups of the alginate and the amino group of chitosan that improves the mechanical resistance of the polymeric network reducing its swelling and erosion at $\mathrm{pH}$ 6.8 . 
TABle 3: Time ( $\mathrm{min}$ ) necessary to release 10,50 , and $90 \%$ of the loaded drug.

\begin{tabular}{ccccc}
\hline & CAl 600 & CAl 400 & CAlCh 600 & CAlCh 400 \\
\hline \multicolumn{5}{c}{ HCl } \\
$t 10 \%$ & 24 & 12 & 31 & 85 \\
$t 50 \%$ & - & - & - & - \\
$t 90 \%$ & - & - & - & - \\
\hline \multicolumn{5}{c}{$\mathrm{pH} 6.8$} \\
$t 10 \%$ & 16 & 19 & 10 & 30 \\
$t 50 \%$ & 130 & 110 & 261 & 310 \\
$t 90 \%$ & 290 & 220 & $>8 \mathrm{~h}$ & $>8 \mathrm{~h}$ \\
\hline \multicolumn{5}{c}{$\mathrm{pH} \mathrm{7.4}$} \\
$t 10 \%$ & 16 & 25 & 28 & 35 \\
$t 50 \%$ & 229 & 187 & 423 & $>8 \mathrm{~h}$ \\
$t 90 \%$ & $>8 \mathrm{~h}$ & 444 & $>8 \mathrm{~h}$ & $>8 \mathrm{~h}$ \\
\hline
\end{tabular}

Surprisingly, the celecoxib release rate slows down mainly for CAlCh 400 formulation even if this formulation is characterized by the lowest particle size. Probably the small diameter and the high surface area of these particles lead to the formation of a thicker chitosan layer around the beads, which opposes a great resistance to the fluid uptake and, as a consequence, to the drug release.

Also at $\mathrm{pH} 7.4$ the differences in the drug release performances can be attributed to the formulation composition rather than to the particle dimensions (Figure 8). All the formulations showed an almost constant drug release rate. There are no differences between CAl 600 and CAl 400 and between CAlCh 600 and CAlCh 400 release curves; when chitosan is in the beads, the drug release rate decreases.

The same conclusions are shown by the analysis of the results of the drug release through the dissolution parameters $(t 10 \%, t 50 \%$, and $t 90 \%)$ (Table 3 ). The differences in the release behavior of the beads are detected also through the dissolution parameters $t 10 \%, t 50 \%$, and $t 90 \%$ and are well evident for the time necessary to release 50 and $90 \%$ of the loaded drug. In phosphate buffer solutions, alginatechitosan beads require longer times to deliver 50 and $90 \%$ of celecoxib compared to alginate beads. Probably the presence of the alginate-chitosan complex provokes a growth of the structural wrinkles, irregularity and complexity of the bead structure, which make the drug liberation difficult.

\section{Conclusions}

The investigated celecoxib loaded alginate and alginatechitosan beads minimize the drug release in acidic environment favoring this process at intestinal $\mathrm{pH}$ (6.8 and 7.4). The experimental results demonstrate that the presence of chitosan in the formulation is responsible for an increase of the resistance of the bead structure and, as a consequence, for a limitation of the bead swelling ability and for a decrease of the drug release rate at neutral $\mathrm{pH}$. Alginate-chitosan beads could be a valuable vehicle of celecoxib for dosage forms useful as adjuvant therapy in patients with familial polyposis and precancerous disease of colon.

\section{Competing Interests}

The authors declare that they have no competing interests.

\section{References}

[1] T. Pongjanyakul and T. Rongthong, "Enhanced entrapment efficiency and modulated drug release of alginate beads loaded with drug-clay intercalated complexes as microreservoirs," Carbohydrate Polymers, vol. 81, no. 2, pp. 409-419, 2010.

[2] W. R. Gombotz and S. F. Wee, "Protein release from alginate matrices," Advanced Drug Delivery Reviews, vol. 31, no. 3, pp. 267-285, 1998.

[3] L. Illum, "Chitosan and its use as a pharmaceutical excipient," Pharmaceutical Research, vol. 15, no. 9, pp. 1326-1331, 1998.

[4] B. D. Ulery, L. S. Nair, and C. T. Laurencin, "Biomedical applications of biodegradable polymers," Journal of Polymer Science Part B: Polymer Physics, vol. 49, no. 12, pp. 832-864, 2011.

[5] M. George and T. E. Abraham, "Polyionic hydrocolloids for the intestinal delivery of protein drugs: alginate and chitosana review," Journal of Controlled Release, vol. 114, no. 1, pp. 1-14, 2006.

[6] C. Ouwerx, N. Velings, M. M. Mestdagh, and M. A. V. Axelos, "Physico-chemical properties and rheology of alginate gel beads formed with various divalent cations," Polymer Gels and Networks, vol. 6, no. 5, pp. 393-408, 1998.

[7] S. K. Bajpai and S. Sharma, "Investigation of swelling/degradation behavior of alginate beads cross-linked with $\mathrm{Ca}^{2+}$ and $\mathrm{Ba}^{2+}$ ions," Reactive and Functional Polymers, vol. 59, no. 2, pp. 129140, 2004.

[8] L. G. Griffith, "Polymeric biomaterials," Acta Materialia, vol. 48, no. 1, pp. 263-277, 2000.

[9] G. T. Grant, E. R. Morris, D. A. Rees, P. J. C. Smith, and D. Thom, "Biological interactions between polysaccharides and divalent cations: the egg-box model," FEBS Letters, vol. 32, no. 1, pp. 195198, 1973.

[10] M. J. Fernández-Hervás, M. A. Holgado, A. Fini, and J. T. Fell, "In vitro evaluation of alginate beads of a diclofenac salt," International Journal of Pharmaceutics, vol. 163, no. 1-2, pp. 2334, 1998.

[11] M. R. Rasmussen, T. Snabe, and L. H. Pedersen, "Numerical modelling of insulin and amyloglucosidase release from swelling Ca-alginate beads," Journal of Controlled Release, vol. 91, no. 3, pp. 395-405, 2003.

[12] Y. Xu, C. Zhan, L. Fan, L. Wang, and H. Zheng, "Preparation of dual crosslinked alginate-chitosan blend gel beads and in vitro controlled release in oral site-specific drug delivery system," International Journal of Pharmaceutics, vol. 336, no. 2, pp. 329337, 2007.

[13] G. A. F. Roberts, Chitin Chemistry, McMillan Press, London, UK, 1992.

[14] A. Fakhry, G. B. Schneider, R. Zaharias, and S. Şenel, "Chitosan supports the initial attachment and spreading of osteoblasts preferentially over fibroblasts," Biomaterials, vol. 25, no. 11, pp. 2075-2079, 2004.

[15] Y.-H. Lin, H.-F. Liang, C.-K. Chung, M.-C. Chen, and H.W. Sung, "Physically crosslinked alginate/N,O-carboxymethyl chitosan hydrogels with calcium for oral delivery of protein drugs," Biomaterials, vol. 26, no. 14, pp. 2105-2113, 2005.

[16] Q. S. Zhao, Q. X. Ji, K. Xing, X. Y. Li, C. S. Liu, and X. G. Chen, "Preparation and characteristics of novel porous hydrogel 
films based on chitosan and glycerophosphate," Carbohydrate Polymers, vol. 76, no. 3, pp. 410-416, 2009.

[17] J. Berger, M. Reist, J. M. Mayer, O. Felt, N. A. Peppas, and R. Gurny, "Structure and interactions in covalently and ionically crosslinked chitosan hydrogels for biomedical applications," European Journal of Pharmaceutics and Biopharmaceutics, vol. 57, no. 1, pp. 19-34, 2004.

[18] J. Berger, M. Reist, J. M. Mayer, O. Felt, and R. Gurny, "Structure and interactions in chitosan hydrogels formed by complexation or aggregation for biomedical applications," European Journal of Pharmaceutics and Biopharmaceutics, vol. 57, no. 1, pp. 35-52, 2004.

[19] K. Y. Lee, W. H. Park, and W. S. Ha, "Polyelectrolyte complexes of sodium alginate with chitosan or its derivatives for microcapsules," Journal of Applied Polymer Science, vol. 63, no. 4, pp. 425-432, 1997.

[20] M. L. González-Rodríguez, M. A. Holgado, C. SánchezLafuente, A. M. Rabasco, and A. Fini, "Alginate/chitosan particulate systems for sodium diclofenac release," International Journal of Pharmaceutics, vol. 232, no. 1-2, pp. 225-234, 2002.

[21] L. Becherán-Marón, C. Peniche, and W. Argüelles-Monal, "Study of the interpolyelectrolyte reaction between chitosan and alginate: influence of alginate composition and chitosan molecular weight," International Journal of Biological Macromolecules, vol. 34, no. 1-2, pp. 127-133, 2004.

[22] A. K. Anal and W. F. Stevens, "Chitosan-alginate multilayer beads for controlled release of ampicillin," International Journal of Pharmaceutics, vol. 290, no. 1-2, pp. 45-54, 2005.

[23] G. Pasparakis and N. Bouropoulos, "Swelling studies and in vitro release of verapamil from calcium alginate and calcium alginate-chitosan beads," International Journal of Pharmaceutics, vol. 323, no. 1-2, pp. 34-42, 2006.

[24] M. L. Huguet and E. Dellacherie, "Calcium alginate beads coated with chitosan: effect of the structure of encapsulated materials on their release," Process Biochemistry, vol. 31, no. 8, pp. 745-751, 1996.

[25] O. Gåserød, O. Smidsrød, and G. Skjåk-Bræk, "Microcapsules of alginate-chitosan-I. A quantitative study of the interaction between alginate and chitosan," Biomaterials, vol. 19, no. 20, pp. 1815-1825, 1998.

[26] O. Gåserød, A. Sannes, and G. Skjåk-Bræk, "Microcapsules of alginate-chitosan. II. A study of capsule stability and permeability," Biomaterials, vol. 20, no. 8, pp. 773-783, 1999.

[27] P.-W. Hsiao, C.-C. Chang, H.-F. Liu, C.-M. Tsai, T. H. Chiu, and J.-I. Chao, "Activation of p38 mitogen-activated protein kinase by celecoxib oppositely regulates survivin and gammaH2AX in human colorectal cancer cells," Toxicology and Applied Pharmacology, vol. 222, no. 1, pp. 97-104, 2007.

[28] M. N. A. Bijman, C. A. Hermelink, M. P. A. van Berkel et al., "Interaction between celecoxib and docetaxel or cisplatin in human cell lines of ovarian cancer and colon cancer is independent of COX-2 expression levels," Biochemical Pharmacology, vol. 75, no. 2, pp. 427-437, 2008.

[29] D. M. Ghorab, M. M. Amin, O. M. Khowessah, and M. I. Tadros, "Colon-targeted celecoxib-loaded Eudragit ${ }^{\circledR}$ S100-coated poly$\varepsilon$-caprolactone microparticles: preparation, characterization and in vivo evaluation in rats," Drug Delivery, vol. 18, no. 7, pp. 523-535, 2011.

[30] Y. Lee, H. Kim, W. Kim, J.-H. Yoon, S. H. Jeong, and Y. Jung, "Colon-specific delivery of celecoxib is a potential strategy to improve toxicological and pharmacological properties of the selective Cox-2 inhibitor: implication in treatment of familiar adenomatous polyposis," Journal of Drug Targeting, vol. 20, no. 6, pp. 524-534, 2012.

[31] Y. S. R. Krishnaiah, V. Satyanarayana, B. D. Kumar, and R. S. Karthikeyan, "Studies on the development of colon-targeted delivery systems for celecoxib in the prevention of colorectal cancer," Journal of Drug Targeting, vol. 10, no. 3, pp. 247-254, 2002.

[32] S. N. Kang, S.-S. Hong, M.-K. Lee, and S.-J. Lim, "Dual function of tributyrin emulsion: solubilization and enhancement of anticancer effect of celecoxib," International Journal of Pharmaceutics, vol. 428, no. 1-2, pp. 76-81, 2012.

[33] M. Rahman, K. Selvarajan, M. R. Hasan et al., "Inhibition of COX-2 in colon cancer modulates tumor growth and MDR-1 expression to enhance tumor regression in therapy-refractory cancers in vivo," Neoplasia, vol. 14, no. 7, pp. 624-633, 2012.

[34] L. Segale, P. Mannina, L. Giovannelli, and F. Pattarino, "Calcium alginate multi-unit oral dosage form for delayed release of celecoxib," Journal of Drug Delivery Science and Technology, vol. 26, pp. 35-43, 2015.

[35] C. Remuñán-López and R. Bodmeier, "Mechanical, water uptake and permeability properties of crosslinked chitosan glutamate and alginate films," Journal of Controlled Release, vol. 44, no. 2-3, pp. 215-225, 1997.

[36] P. Costa and J. M. Sousa Lobo, "Modeling and comparison of dissolution profiles," European Journal of Pharmaceutical Sciences, vol. 13, no. 2, pp. 123-133, 2001.

[37] Food \& Drug Administration, Guidelines for IndustryDissolution Testing of Immediate Release Solid Oral Dosage Forms, Food \& Drug Administration, 1997.

[38] N. D. Ceki, J. R. Milić, S. D. Savić, M. M. Savić, Z. Jović, and R. Daniels, "Influence of the preparation procedure and chitosan type on physicochemical properties and release behavior of alginate-chitosan microparticles," Drug Development and Industrial Pharmacy, vol. 35, no. 9, pp. 1092-1102, 2009. 

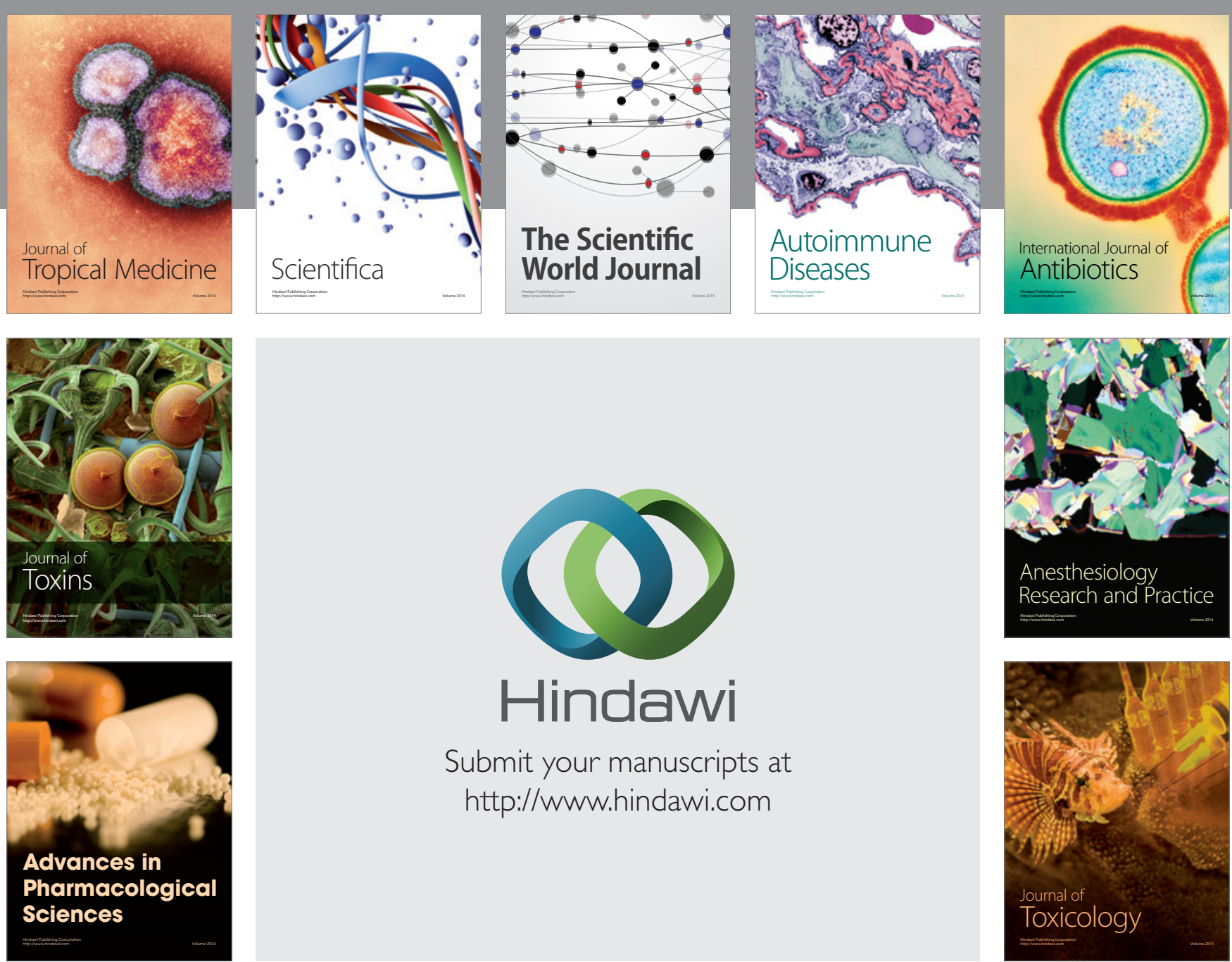

\section{Hindawi}

Submit your manuscripts at

http://www.hindawi.com
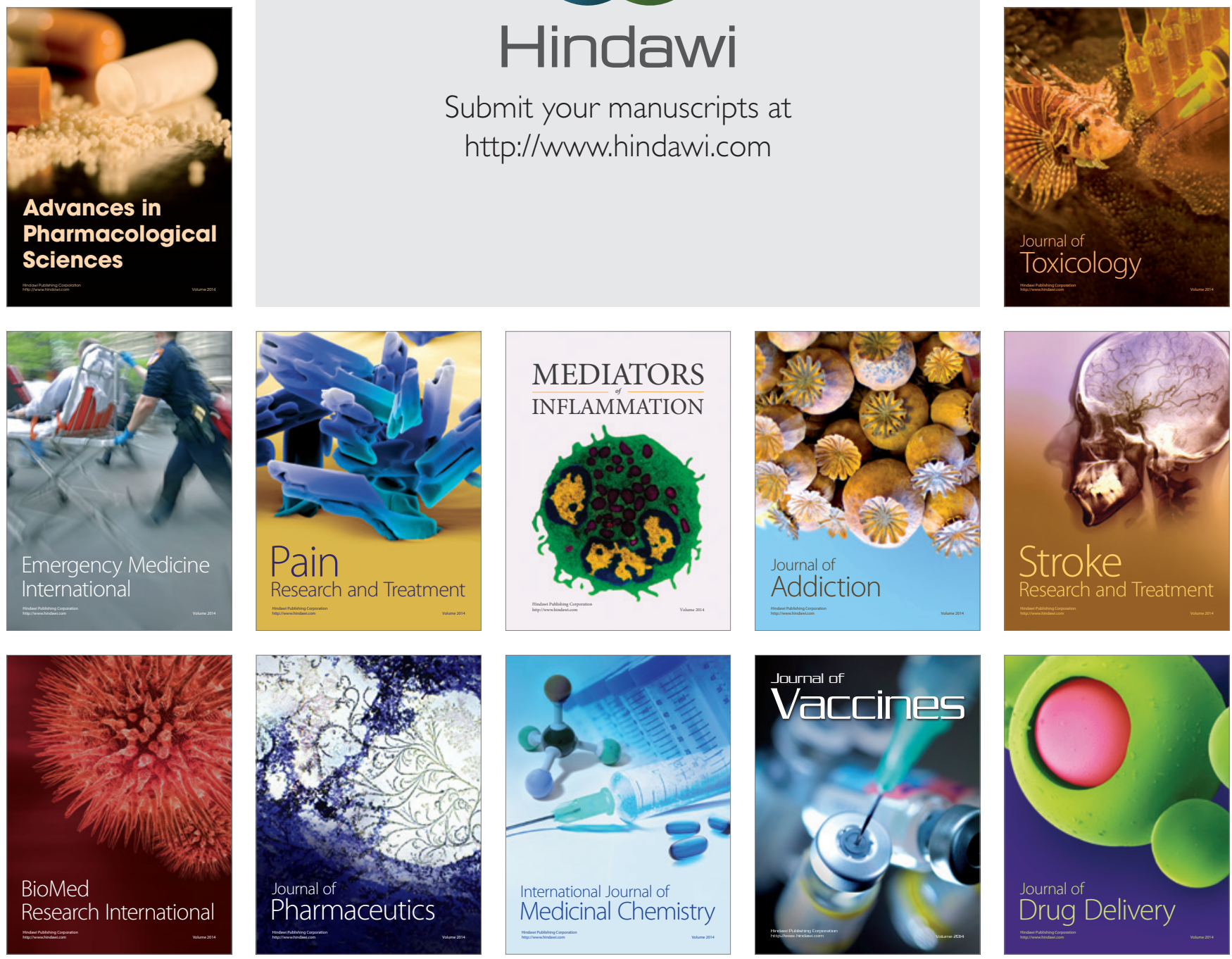